\title{
The Acceleration History of the Universe and the Properties of the Dark Energy
}

\author{
Ruth A. Daly* and S. G. Djorgovski ${ }^{\dagger}$ \\ ${ }^{*}$ Department of Physics, Penn State University, Berks Campus, P. O. Box 7009, Reading, PA 19610 \\ ${ }^{\dagger}$ Division of Physics, Mathematics, and Astronomy, Caltech, MS 105-24, Pasadena, CA 91125
}

\begin{abstract}
The model-independent method of using type Ia supernovae proposed and developed by Daly \& Djorgovski $(2003,2004)$ has been applied to the Riess et al. (2007) supernovae sample. Assuming only a Robertson-Walker metric, we find $q_{0}=-0.5 \pm 0.13$, indicating that the universe is accelerating today. This result is purely kinematic, is independent of the contents of the universe, and does not require that a theory of gravity be specified. Our model-independent method allows a determination of $q(z)$ for a particular value of space curvature. When $q(z)$ transitions from negative to positive values, the universe transitions from an accelerating to a decelerating state. For zero space curvature, we find that the universe transitions from acceleration to deceleration at a zedshift of about $z_{T}=0.35_{-0.7}^{+0.15}$ for the Riess et al. (2007) sample.

If a theory of gravity is specified, the supernovae data can be used to determine the pressure, energy density, and equation of state of the dark energy, and the potential and kinetic energy density of a dark energy scalar field as functions of redshift. The relevant equations from General Relativity are applied, and these functions are obtained. The results are consistent with predictions in the standard Lambda Cold Dark Matter model at about the two sigma level.
\end{abstract}

Keywords: Cosmology

PACS: $98.80-\mathrm{k}$

\section{INTRODUCTION}

Determination of the expansion history of the universe, from which we can constrain the physical nature of its matter and energy constituents, is the central problem of traditional cosmology. One way it can be addressed is through the use of a set of coordinate distances and redshifts for some standard set of objects. Type Ia supernovae provide a modified standard candle (e.g. [1,2]) that allow the distance modulus, luminosity distance, and coordinate distance to each source to be determined.

In a novel, model-independent approach to this problem, it was shown by [3] that the first and second derivatives of the coordinate distance with respect to redshift could be obtained from the coordinate distances and combined to solve for the expansion rate $H(z) / H_{0}$ and acceleration rate $q(z)$ of the universe. The only assumptions are that the universe is described by a Robertson-Walker metric and has zero space curvature. The results are independent of the contents of the universe and the properties of these contents, and independent of whether General Relativity provides an accurate description of the universe. The assumption of zero space curvature is dropped by [4], who show that the deceleration parameter at a redshift of zero, $q_{0}$, remains the same, independent of whether space curvature is zero or non-zero.

To study the properties of the dark energy as a function of redshift, the first and second

CP937, Supernova 1987A: 20 Years After, edited by S. Immler, K. Weiler, and R. McCray (C) 2007 American Institute of Physics 978-0-7354-0448-9/07/\$23.00 

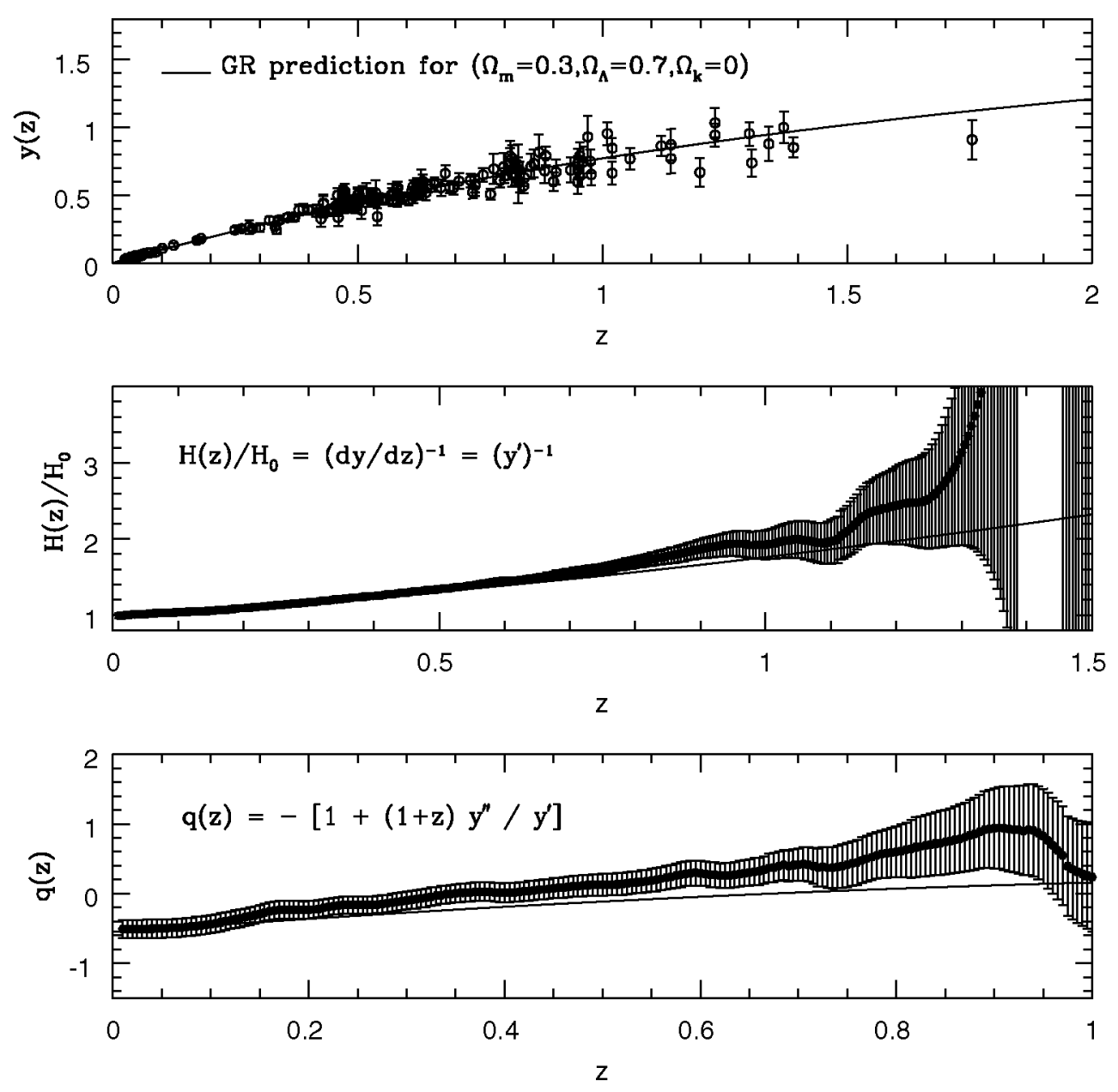

FIGURE 1. Dimensionless coordinate distances to 182 type Ia supernovae of [6], obtained with the best fit parameters presented by [7], are shown in the top panel. A window function of width 0.6 in redshift was used to obtain the first and second derivatives of the coordinate distance with respect to redshift, as described by $[3,5]$, and these are used to determine the expansion and acceleration rates of the universe as functions of redshift, shown in the second and third panels.

derivatives of the coordinate distance can be combined to solve for the pressure, energy density, equation of state, and kinetic and potential energy densities of the dark energy if a theory of gravity be specified, as discussed by [5]. Einstein's Equations from General Relativity for an isotropic and homogeneous universe are used to define the relationship 


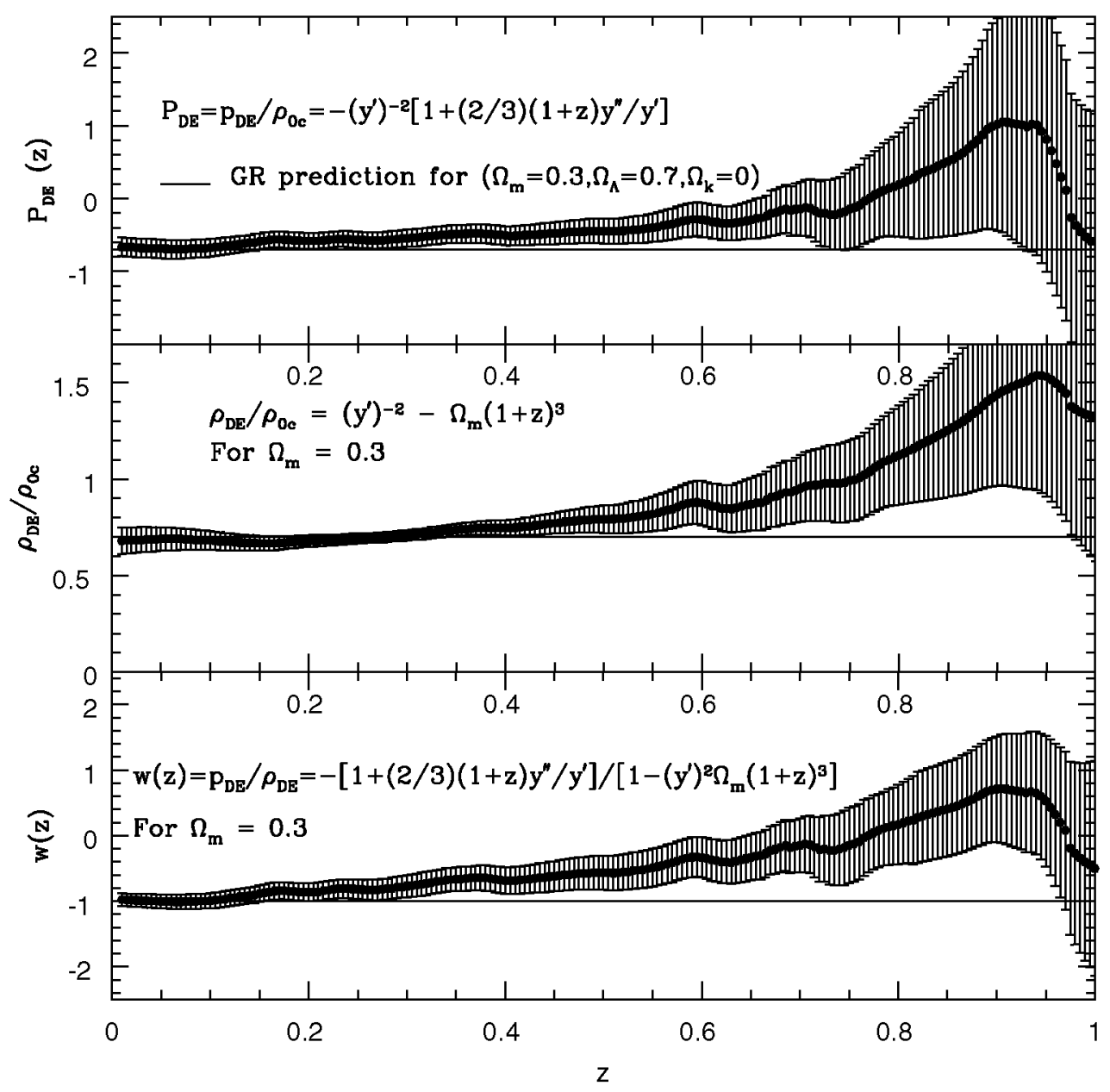

FIGURE 2. The pressure, energy density, and equation of state of the dark energy obtained by combining the first and second derivatives of the coordinate distance for the sample of [6]. As described by [5], a theory of gravity must be specified to obtain the pressure of the dark energy as a function of redshift, and General Relativity has been applied here. To obtain the energy density and equation of state of the dark energy as functions of redshift, the present value of the mean mass density in non-relativistic matter must be specified, and we have adopted $\Omega_{m}=0.3$.

between the dark energy pressure and energy density, the mass-energy density of nonrelativistic matter, and the expansion and acceleration rates of the universe. Given that the expansion and acceleration rates of the universe are known independent of the 


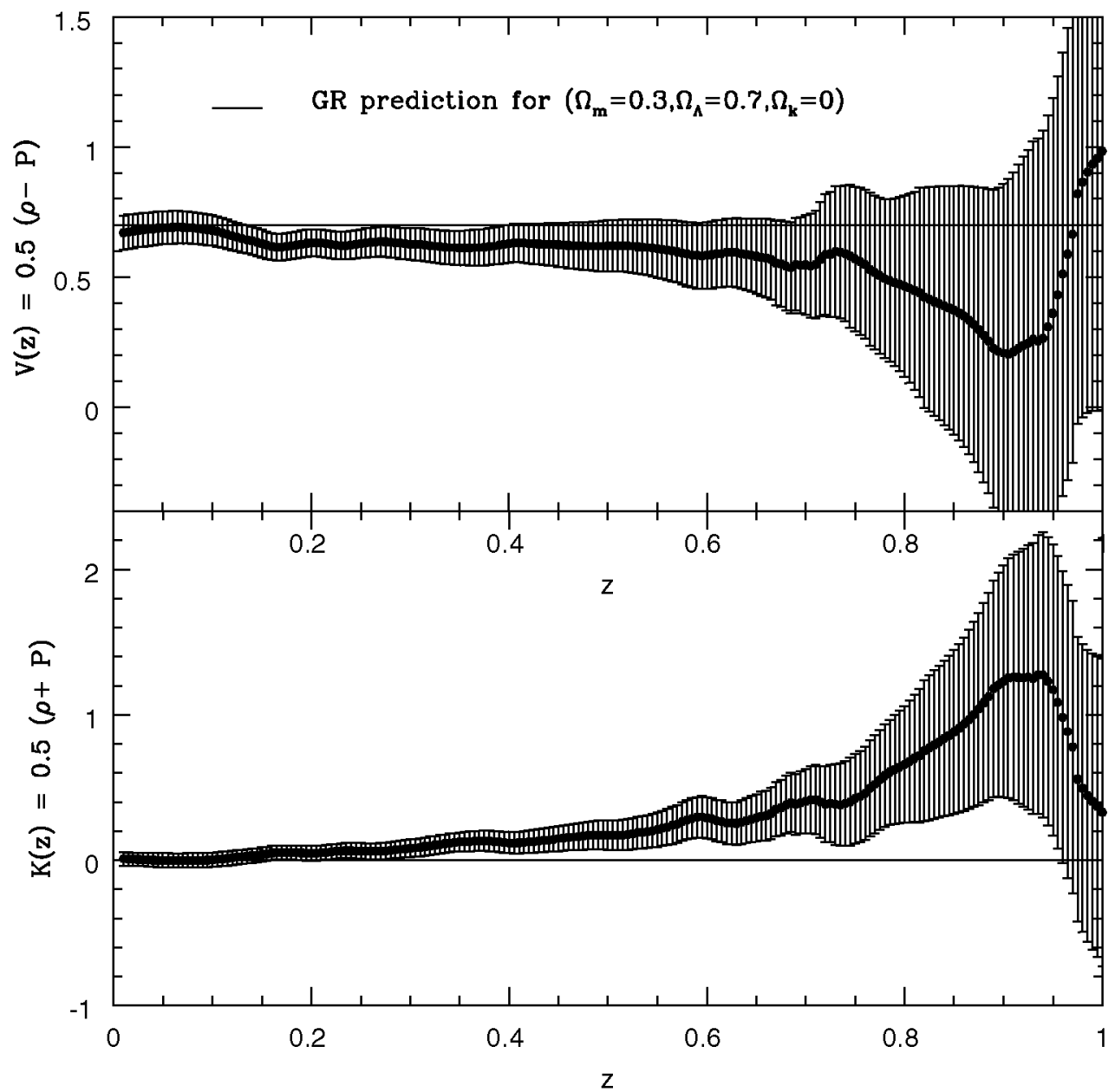

FIGURE 3. The kinetic and potential energy densities of a dark energy scalar field can be obtained by combining the pressure and energy density of the dark energy. These are shown here for the supernovae sample of [6].

contents of the universe, these equations allow a determination of the pressure, energy density, and equation of state of the dark energy as functions of redshift, as shown by [5], and the kinetic and potential energy density of the dark energy. 


\section{SUMMARY}

Our model-independent method has been applied to the $182 \mathrm{SN}$ presented by [6]. The values of the coordinate distance to each source are obtained from the distance moduli, as described by [7].

As shown in Figure 1, the current value of the acceleration parameter is $q_{0}=$ $-0.5 \pm 0.13$. This indicates that the universe is accelerating today with greater than $3 \sigma$ confidence. Note that our determination of $q_{0}$ depends only upon the assumption that the universe is described by a Robertson-Walker metric, and is independent of the value of space curvature, as discussed in detail by [4], and as is clear from the equations presented by [3].

The redshift of transition from a state of acceleration to a state of deceleration does require that a value of space curvature be specified, so two assumption are made to determine $z_{T}$ : zero space curvature and a Robertson-Walker line element. For the sample of [6], we find $z_{T}=0.35_{-0.7}^{+0.15}$.

The first and second derivatives of the coordinate distance with respect to redshift are combined to solve for the pressure, energy density, and equation of state of the dark energy as functions of redshift by applying the equations derived by [5]. The results are shown in Figure 2. The first and second derivatives of the coordinate distance can also be combined to obtain the kinetic and potential energy density of a dark energy scalar field, and these results are shown in Figure 3.

Overall, the results are consistent with those expected in a standard Lambda Cold Dark Matter model, in which a cosmological constant is responsible for the acceleration history of the universe, at about the two sigma level. Further details of this study are presented by [4].

\section{ACKNOWLEDGMENTS}

We would like to thank the observers for their tireless efforts to obtain the supernovae data that makes these studies possible. This work was supported in part by U. S. National Science Foundation grants AST-0507465 (R.A.D.) and AST-0407448 (S.G.D.), and the Ajax Foundation (S.G.D.).

\section{REFERENCES}

1. M. M. Phillips, $A p J, 413, \mathrm{~L} 105$ (1993).

2. M. Hamuy, M. M. Phillips, J. Maza, N. B. Suntzeff, R. A. Schommer, \& R. Aviles, $A J, 109,1$ (1995).

3. R. A. Daly, and S. G. Djorgovski, $A p J$, 597, 9-20 (2003).

4. R. A. Daly, S. G. Djorgovski, K. Freeman,M. P. Mory, C. P. O’Dea, P. Kharb, \& S. Baum, submitted (2007).

5. R. A. Daly, and S. G. Djorgovski, $A p J, 612,652-659$ (2004).

6. A. Riess, et al., $A p J, 659,98-121$ (2007).

7. R. A. Daly, M. P. Mory, C. P. O’Dea, P. Kharb,S. Baum, \& E. J. Guerra, submitted (2007). 